\title{
EXPLORANDO LA CONSTRUCCIÓN DE LA SUBJETIVIDAD LABORAL: DEL EJECUTOR DE OFICIOS, AL TRABAJADOR PSICOLOGIZADO ${ }^{1}$
}

\section{The construction of labour subjectivity: from the tradesman, to the psychological worker}

\author{
Hernán Camilo Pulido Martínez ${ }^{2}$ \\ Marietta Mejía de Mesa ${ }^{3}$ \\ Pontificia Universidad Javeriana
}

DOI: 10.17533/udea.rp.v10n1a10

Recibido: 2017-12-01 Aceptado: 2018-02-15

Para citar este artículo en APA: Pulido, H., y Mejía, M. (2018). Explorando La construcción de la subjetividad laboral: del ejecutor de oficios, al trabajador psicologizado. Revista de Psicología Universidad de Antioquia, 10 (1), 237-264. DOI: 10.17533/udea.rp.v10n1a10

Resumen: El artículo presenta una serie de figuras de la subjetividad laboral que emergieron en un hospital universitario entre los años 1942 y 2014. Se considera el surgimiento de estas figuras a la luz de: las transformaciones de las condiciones laborales, los cambios geopolíticos en los modelos del ejercicio de la medicina, el desarrollo de la psicología, así como de las prescripciones nacionales e internacionales para la regulación del trabajo. Los materiales que se utilizaron provinieron de estudios que consideraban o describían diversos aspectos para el manejo de los trabajadores en el hospital. Los resultados muestran la relación entre las concepciones acerca de cómo los trabajadores son y cómo deben actuar antes y después de la introducción de la psicología académica.

Palabras clave: psicología del trabajo, subjetividad, regulación, producción, gobierno.
Abstract: The article presents a series of figures of labour subjectivity that emerged in the context of a university hospital between 1942 and 2014. The emergence of these figures is considered in the light of: transformations of working conditions, geopolitical changes in the models of the practice of medicine, development of psychology, as well as national and international prescriptions for the regulation of work. The materials that were used came from studies that considered or described various aspects for the management of workers in the hospital. The results show the relationship between conceptions about how workers are and how they should act before and after the introduction of academic psychology.

Keywords: work psychology, subjectivity, regulation, production, government.

1 Este artículo es resultado de investigación del proyecto "La relación psicología-trabajo en organizaciones del sector de la salud: aportes para la reconstrucción histórica de la subjetividad en el mundo del trabajo" (ID: 00004234), financiado por la Vicerrectoría Académica de la Pontificia Universidad Javeriana, Bogotá.

2 Doctor en Psicología, Universidad de Cardiff (Reino Unido), Magíster en Comunicación y

Cultura y Psicólogo, Pontificia Universidad Javeriana. Profesor Titular Facultad de Psicología. Correo electrónico: cpulido@javeriana.edu.co; http://orcid.org/0000-0003-1071-2784.

3 Magíster en Historia, Universidad Pontificia Javeriana. Correo electrónico: mariettamejiadem@ yahoo.com; http://orcid.org/0000-0003-2922-3323. 


\section{Introducción}

Hoy en día resulta corriente que las estrategias de corte psicológico estén colonizando nuevos ámbitos laborales. Ya nadie se extraña de que esto ocurra. Por el contrario, se celebra que la psicología se expanda fácilmente en el amplio espectro de escenarios que hacen parte del mundo laboral (Pulido Martínez, 2007; Staeuble, 2005). Usualmente se considera que en aquellas organizaciones donde ya están siendo aplicadas las estrategias psicológicas, nuevas intervenciones deban ser formuladas para apoyar la gerencia en su objetivo de aumentar la productividad. No solo en las organizaciones se presentan herramientas psicológicas de muy diversos tipos para administrar la fuerza de trabajo; también en las librerías se ofrecen best sellers con fórmulas milagrosas para ser aplicadas en los ámbitos laborales. Al mismo tiempo, las consultorías nacionales e internacionales hacen sus intervenciones basadas en conocimientos psicológicos, mientras las gerencias hacen uso de los discursos y prácticas psicológicas, como parte del arsenal de herramientas para la administración de los recursos humanos. Como ha sido señalado en la literatura, la empresa psicológica no se detiene, a tal punto que actualmente tanto el trabajo como los trabajadores y las organizaciones son entendidos desde algún tipo de psicología (Hollway, 1991).

En este panorama, sin embargo, son relativamente pocos los estudios que se han preocupado por establecer el proceso mediante el cual la psicología gana un lugar en los ámbitos laborales particulares (Dávila Ladrón de Guevara, 2005). A esas escasas investigaciones bien les cabe el rótulo de críticas, en la medida en que se apartan del interés por promocionar la eficiencia empresarial, propio de la psicología industrial-organizacional. Antes bien, estos estudios se alejan de dar respuesta a la pregunta típica de la administración: “¿Cómo lograr una mayor productividad?”, para concentrarse en resolver interrogantes relacionados con el proceso por medio del cual las prescripciones de la psicología se instauraron en las organizaciones laborales. (Castaño \& Sánchez, 1978; Crespo \& Serrano Pascual, 2013; Hollway, 1991; Pulido Martínez, 2010, 2017; Pulido Martínez \& Carvajal Marín, 2016).

Consideraciones particulares merecen las investigaciones referidas al ma- 
nejo de la fuerza laboral en las organizaciones del sector de la salud. En este caso, por una parte, se reconoce que la eficiencia organizacional depende de un adecuado manejo de los recursos humanos con los que se cuenta. Sin embargo, por otra parte, la mayoría de las veces se ha dejado de lado la investigación en esta área. Tal como señalan Townsend y Wilkinson (2010), esta carencia en la investigación es muy grave en las organizaciones que ofrecen servicios de salud, porque se le pide al hospital que sea cada día más eficiente, pero no se considera, en términos investigativos, que los recursos humanos sean considerados como un punto indispensable para alcanzar esta meta. En esta misma línea, se puede afirmar que si bien la psicología ha sido aplicada en los hospitales desde hace muchas décadas (Marcon, Luna \& Lisboa, 2004; Pereira da Silva, 2006), los investigadores no han llevado a cabo estudios de corte crítico que indaguen por las formas en las que este conocimiento y sus prácticas derivadas han llegado a ocupar un lugar preponderante en la administración de la fuerza laboral.

A partir de estas consideraciones, a continuación se presenta un mapeo exploratorio e ilustrativo — no exhaustivo— de la producción de figuras de la subjetividad que se utilizaron entre los años 1942 y 2014, en un Hospital Universitario del más alto nivel de complejidad para la atención de la salud en Bogotá (Colombia). Como ha sido discutido ampliamente en la literatura, estas figuras de la subjetividad tienen una importancia capital, porque, con base en ellas, diferentes autoridades han llevado a cabo acciones que les han permitido ejercer el gobierno de los trabajadores en las organizaciones donde se labora (Foucault, 2006; García-Álvarez, 2008; Hollway, 1991; Miller \& Rose, 2008; Pulido-Martínez, 2007; Rose, 1996).

\section{La psicología y las formas de la subjetividad en el mundo laboral}

Las críticas que se le han formulado a la relación que la psicología ha establecido con el mundo del trabajo son muchas y muy variadas. Por ejemplo, son bien conocidas las objeciones que se han propuesto tanto respecto de su 
sesgo gerencial (Baritz, 1960) como de su carencia de conceptos para entender el mundo del trabajo y, por tanto, para hacer aportes teóricos relevantes (Fernández Ríos, 1995; Gordon, Kleiman \& Hanie, 1978; Lamal, 1991). Igualmente, se ha criticado el carácter ideológico que asume la psicología cuando entra en relación con el mundo del trabajo, al impedir ver las relaciones de clase en los sitios donde se labora (Bramel \& Friend, 1981), así como se ha señalado el "olvido" que esta disciplina ha demostrado por algunas organizaciones de trabajadores, como los sindicatos, al tiempo que se le ha acusado de promover una visión particular de los trabajadores, como sujetos fácilmente manipulables (Dávila Ladrón de Guevara, 2001; Huszczo, Wiggins \& Currie, 1984; Prilleltensky, 1994). En general, estas formas de examinar la psicología se encargan de mostrar cómo el conocimiento psicológico y las prácticas que de él se derivan de muy variadas maneras trivializan, distorsionan, o manipulan el mundo del trabajo, al olvidar o dejar de lado las condiciones laborales como foco central de análisis. Entonces, este tipo de críticas, al considerar las operaciones que hace la psicología en relación con el trabajo, abren el camino hacia la búsqueda de propuestas para ejercer una mejor práctica, que supere los inconvenientes que se le señalan (Parker, 2007; Pulido-Martínez \& Sato, 2013; Rose, 1996).

Otras miradas sobre la relación entre la psicología y el trabajo se alejan de estas perspectivas que buscan construir una psicología más adecuada, para concentrarse en examinar el lugar de este conocimiento en las sociedades contemporáneas (Hollway, 1991; Pulido Martínez, 2014; Rose, 1996). Se retoma aquí como argumento central el que la psicología tiene una injerencia fundamental en la producción de la subjetividad de los trabajadores (Hollway, 1991; Townley, 1994). Se asume, entonces, que mediante los discursos y prácticas psicológicas se produce un efecto, por ejemplo, de individualización y, a la vez, de homogeneización de los trabajadores, de modo tal que se pueden ejercer unas acciones de gobierno, de acuerdo con las características particulares de los trabajadores y de los grupos a los cuales pueden pertenecer (Henriques, Hollway, Urwin, Venn \& Walkerdine, 1998; Holmer, 1997). En esta perspectiva de la crítica, la psicología también provee los parámetros con los cuales los trabajadores y las organizaciones van a ser comparados y rearticulados, para 
llevar a cabo una forma de gobierno "a distancia". De este modo, se transforma el papel del Estado, al dejar la conducción de los trabajadores en manos de las organizaciones laborales, lugar en donde se ponen en práctica las racionalidades de gobierno imperantes (Miller \& Rose, 2008; Pulido Martínez, 2010).

Ahora, si bien las investigaciones sobre las operaciones de la psicología respecto a la producción de la subjetividad en los países donde no se producen los objetos psicológicos han señalado que estas críticas son pertinentes para todos aquellos ámbitos en donde la psicología se hace presente (Pulido Martínez, 2017a), también señalan que estas maneras de analizar las funciones que cumple la psicología tienen ciertas limitaciones, especialmente cuando se consideran las condiciones locales. Quizá, la más importante de las objeciones formuladas a las críticas mencionadas es la relativa a su carácter universalista, el cual supone que en todos y cada uno de los ámbitos laborales a los que llega la psicología se producirán los mismos efectos de subjetividad, las mismas formas de gobierno e idénticas consecuencias en su aplicación. Para tratar de salir de este problema universalista, los investigadores reclaman que se recupere la dimensión local de la crítica referida a las relaciones de poder (subyacentes a la constitución del conocimiento) entre los países productores y consumidores de la psicología. No obstante, lo que se reclama en esta perspectiva no es la adaptación cultural de los instrumentos psicológicos para que se tornen pertinentes y puedan ser aplicados en las organizaciones laborales locales; al contrario, con ello lo que se busca es examinar cómo la diseminación de la psicología tiende a reproducir relaciones de subordinación, por ejemplo, cuando a través de procesos de ajuste cultural, reafirma la validez de la ciencia producida en otros contextos (Pulido Martínez, 2017b, 2018).

En particular, en lo que se refiere al trabajo y las organizaciones laborales, el argumento que se esgrime desde esta crítica geopolítica del conocimiento se presenta de la siguiente manera: el conocimiento psicológico, sus prácticas e instrumentos han sido diseñados para resolver problemas específicos del ámbito laboral en los lugares donde se produce la psicología; entonces cuando se procura la aplicación de estos conocimientos, prácticas e instrumentos en otros puntos del planeta, los problemas locales del trabajo se subordinan ante la aplicación del producto foráneo. Esta situación implica que los problemas 
del mundo laboral no se consideren en sus dimensiones (sociales, culturales, económicas e históricas) particulares, sino que se los aparte, sin ser examinados (Castaño \& Sánchez, 1978).

Al menos tres caminos alternativos surgen de esta situación. El primero le da espacio a la psicología, con la intención de hacerla pertinente para las condiciones locales. Este camino de hacer una psicología del trabajo autóctona y relevante para regiones como América Latina ya cuenta con algún recorrido (cf. Bendassolli, 2013; Bernardo, Oliveira, Sousa, \& Sousa, 2017; Coutinho, Borjes, Graf \& Da Silva, 2013; Hespanhol Bernardo, 2014; Sato, 2007; Sisto, 2009; Soto, 2008; Stecher, 2014). El segundo propone establecer las condiciones en las cuales se hace posible la relación entre la psicología y la constitución de la subjetividad, para involucrar a los trabajadores del sur global como aquel otro que hace parte del pensar psicológico universal (Flórez Flórez, 2002; Pulido Martínez, 2007, 2017). El último camino, propone la constitución de otro tipo de conocimiento sobre el mundo del trabajo, que no necesariamente tuviera que mantenerse dentro de los límites de lo que se considera psicología (García Ramos, Baez Lebrón, Diaz Juarbe \& Santiago Estrada, 2014).

En todo caso, los estudios sobre las relaciones internacionales que se establecen "gracias a" y "en razón de" la aplicación de la psicología en las organizaciones alrededor del mundo conllevan el problema de la constitución del sujeto psicológico. Si la constitución del sujeto psicológico laboral no se produce de manera inmediata, pueden considerarse, entonces, casos particulares para develar, por ejemplo, cómo la psicología viaja y se establece en lugares específicos, cómo se instauran diversas formas psicológicas de concebir al trabajador y cuáles son las implicaciones particulares de este proceso. Para llevar a cabo esta tarea, se puede partir del efecto de reflexividad que tiene la psicología. Este efecto actúa directamente como productor de subjetividad; es decir, cuando la psicología habla del sujeto que estudia, al mismo tiempo lo produce. En otras palabras, cuando la psicología considera a los trabajadores, al mismo tiempo resulta conformando los tipos de obrero, de empleado, de trabajador autónomo, de colaborador motivado o de desempleado de los cuales habla. Estos trabajadores psicologizados se construyen alrededor de ha- 
bilidades, competencias, rasgos de personalidad, coeficientes de inteligencia, formas de relacionarse, motivaciones intrínsecas y extrínsecas, liderazgos particulares, empleabilidades y demás objetos psicológicos.

Así como no se reproducen espontáneamente esos efectos de subjetividad (Pulido Martínez, 2018; Richards, 2002), tampoco, cuando la psicología viaja alrededor del mundo, llega a terrenos vacíos. Por el contrario, la psicología llega a distintos lugares y se encuentra con las construcciones particulares de lo que los seres humanos son y lo que estos deben hacer. Este encuentro ha dado lugar a diversas formas de concebir las mediaciones que ocurren. Estas mediaciones han sido descritas en la literatura psicológica como emulación (Blowers, Chenung \& Ru, 2009), adaptación (Louw \& Danziger, 1990), indigenización (Danziger, 2006), aculturación (Schwartz, Unger, Zamboanga \& Szapocznik, 2010; Yoon et al. 2013) e hibridación (Gorbach \& López, 2008). Al tener en cuenta que se producen estos procesos de encuentro cuando la psicología se expande alrededor del mundo, es necesario desplazarse de la visión que tradicionalmente ha tenido la psicología industrial-organizacional, la cual se limita a considerar las condiciones en las que se labora más como un contexto, que como una parte fundamental del análisis, para pasar a considerar una perspectiva que tome las condiciones objetivas de trabajo como un asunto central, en la medida en que estas permiten comprender el "terreno abonado" donde la psicología es útil como un saber que sirve para administrar a los trabajadores. De este modo, para comprender el lugar y las operaciones de la psicología, es necesario indagar por las formas que toma la subjetividad en las condiciones de trabajo que estaban presentes antes de que la psicología académica se hiciera presente, así como las cambiantes condiciones laborales en las cuales fue posible que este conocimiento se aplicara. Es en la interacción entre estas formas de subjetividad propuesta por la psicología académica y, podría decirse, las formas locales de concebir a las personas, especialmente en lo que concierne a ser un trabajador, en donde emerge el sujeto laboral psicologizado.

Los análisis que se presentan a continuación se ubican en esta perspectiva. En ellos se muestran las formas de la subjetividad que han emergido en un Hospital Universitario, a lo largo de su historia. Se trata de una manera de 
analizar la trayectoria por medio de la cual la psicología, también ubicada en la periferia, llega a constituirse como el saber que impera al momento de entender el trabajo y los trabajadores. Es una manera de acceder a la constitución de la subjetividad en donde se juntan, como lo sugiere Louw (2006), la psicología y el medio social amplio, para dar como resultado el análisis de "un aspecto central de la disciplina y de su relación con los seres humanos que somos" (Louw, 2006, p. 16).

\section{Metodología}

Las formas de manejo del personal que aquí se presentan provienen de investigaciones históricas y administrativas previas que tuvieron como objeto específico el hospital universitario referido. El desarrollo histórico del hospital, reconstruido a partir de los documentos considerados, se mostró como escenario privilegiado para el estudio de las formas que asume la subjetividad, tanto en relación con la psicología académica, como con las maneras de concebir a los trabajadores anteriores a la introducción del conocimiento psicológico. En esta línea, el hospital universitario se constituyó en un contexto local para observar aquello que ha sido descrito por investigadores en campos como la historia y en los estudios sociales de la psicología, en referencia al encadenamiento que se presenta entre la psicología académica y las formas de concebir a las personas que circulan corrientemente en la sociedad (Danziger, 1997; Martin, 2007; Richards, 2002; 1997; Ward, 2002). En la práctica estas formas de concebir a los seres humanos se hallan en una relación de mutua y constante retroalimentación (Danziger, 1997; Richards, 2002).

El periodo de la indagación entre los años 1942 y 2014 se estableció en relación directa con los orígenes de la institución. En efecto para el año 1942 el hospital ya estaba concebido como tal, si bien con un sinnúmero de carencias financieras que lo tuvieron casi como una entidad inexistente, con un mínimo funcionamiento, motivo por el cual por varios años tuvo sedes "prestadas" para sus prácticas docentes, investigativas y asistenciales, al tiempo que disponía de un enorme potencial humano que gradualmente le permitiría 
desarrollarse hasta llegar a convertirse en una de las instituciones líderes en la atención de pacientes, comprometida con la formación de nuevos profesionales de la salud en el país (Mejía de Mesa, 2014).

Es necesario resaltar aquí que los desplazamientos en las estrategias para el manejo de la fuerza laboral no se conciben, solamente, como una cuestión particular del hospital, o una concreción de voluntades por parte de aquellos que en determinado momento pasaron por la institución. Más bien, este tipo de mudanzas muestran cómo en su base se encuentra una compleja constelación de aspectos, entre los que se cuentan las transformaciones en las lógicas organizacionales (Arndt \& Bigelow, 2013; Eslava, 2002; Ortiz Martínez, 2016); el estado local de la psicología y de la recepción en organizaciones específicas (Harris, Cortvriend \& Hyde, 2007; Pulido Martínez, 2010; Richards, 2002); la geopolítica del conocimiento (Brock, 2006; Steuble, 2005); las pautas y discursos administrativos que prescriben cuáles son las formas correctas de gobernar a los trabajadores (Rose, 1999).

\section{El hospital y las formas de subjetividad}

Se presentan a continuación cinco ficciones de la subjetividad que en forma gradual se fueron estableciendo a lo largo de la historia de la institución. Estas propuestas sobre la subjetividad no se desenvuelven como asuntos abstractos de significación, sino que, por el contrario, las imágenes construidas acerca de cómo son y cómo actúan los trabajadores tienen referentes reales. Por una parte, son el resultado de formas específicas de administrar y, por tanto, de regular a los trabajadores; por otra, a partir de estas, se conciben nuevas formas de manejo de la fuerza laboral, de acuerdo con las condiciones de trabajo imperantes (Pulido-Martínez, 2010; Rose, 1999).

Inicialmente, debe precisarse que el hospital universitario surgió en un medio permeado por instituciones religiosas, en 1942, y que, en un lapso relativamente corto, dejó de concebírselo como una institución que prestaba servicios de caridad, para asumir otra forma más moderna, que ya exigía prescripciones administrativas, con el objeto de transformar su funcionamiento 
tradicional en un estilo empresarial. Se presentan así, por un lado, las "huellas" organizacionales que se le imprimieron al hospital en su fundación y, por otro lado, la nueva imagen, que coincide con la que hoy en día se concreta en torno a la misión organizacional. Es justamente en este lapso comprendido entre las dos formas de concebir al hospital (una relacionada con una mirada filantrópica y educativa, y otra basada en el lenguaje empresarial) donde el conocimiento psicológico y sus prácticas derivadas fueron ganando un importante terreno y adelantaron ciertas operaciones dentro de la institución.

De acuerdo con Mejía de Mesa (2014), en los estatutos fundantes de este hospital, el Consejo Directivo de la Facultad de Medicina formulaba lo que ahora podría considerarse como las primeras normas administrativas generales para la institución. Cuatro ejes resultaban centrales: el fin, el patrimonio, el gobierno y la administración, con los cuales se les daba vía libre a los nombramientos de cargos y funciones. Con el Decreto n. ${ }^{\circ} 22$ del 1 de abril de 1942, mediante el cual se le otorgaba la personería jurídica de carácter religioso al hospital, se establecía que el fin del hospital sería: "practicar la caridad cristiana, dando a los pobres que de toda la República acuden a la capital, asistencia médica gratuita, y servir de campo de estudio, práctica e investigación a los profesores y estudiantes" (Consejo Directivo de la Facultad de Medicina, 1942).

En un marcado contraste con esta visión de lo que debía ser el hospital, en la plataforma estratégica que hoy guía las acciones administrativas del mismo hospital, se establece que: "Somos un hospital universitario que brinda servicios integrales de excelente calidad comprometidos con la conservación del medio ambiente... Damos a nuestros pacientes y sus familias atención humana, ética y segura. Actuamos inspirados en los principios de fraternidad cristiana". También se señala que el hospital quiere ser "auto-sostenible y líder en la prestación de servicios de salud de excelente calidad" (Hospital Universitario San Ignacio, 2014)

Así, de una mirada que consideraba los fines del hospital vinculados ante todo a la atención a los pobres y al servicio de la educación, se pasó, en el transcurso de décadas, a concebir el hospital a partir de la jerga de la calidad, la autosostenibilidad y el liderazgo. Además, se dio un desplazamiento de la atención de una población en situación de vulnerabilidad, a un asunto abstracto de pacientes y familias que deben ser atendidas en términos del hu- 
manismo, la ética y el riesgo. De este tipo de transformaciones, que ha sido descrito en la literatura como la transición desde el modelo de A. Flexner a los centros académicos de salud (Ortiz Martínez, 2016), no han sido consideradas suficientemente las implicaciones respecto al manejo de la fuerza laboral.

$\mathrm{Al}$ inaugurarse el hospital, según Mejía de Mesa (2014), las religiosas de La Presentación de Tours, provenientes de Francia, estaban a cargo de la administración de la institución, quienes, en su particular organización jerárquica dentro de la comunidad, entrenaban y supervisaban al personal "paramédico". El síndico del hospital (también perteneciente a una comunidad religiosa), era también central, y representaba un prototipo de la administración, que después sufriría grandes cambios. De acuerdo con Mejía de Mesa (2014), al síndico le correspondía hacerse cargo de una serie de actividades relacionadas con distintos actores sociales que contribuían a mantener financieramente la institución, y tenía el deber de "conservar y cuidar el archivo de las posesiones, de la administración y de la contabilidad del Hospital” (p. 132). El papel de las religiosas de La Presentación también se destaca por depender de una relación diaria y directa con la administración y la educación de los trabajadores, en momentos en los que, tanto en la sede provisional como en la luego definitiva sede del hospital, se vivían condiciones económicas muy precarias.

Mejía de Mesa (2014) hace referencia a un tipo particular de gobierno hospitalario que le fue encomendado originalmente a las religiosas de La Presentación de Tours. Desde unas relaciones administrativas de carácter "monástico", sus roles permitían establecer una concepción de lo que sería un trabajador al que se le pudiera denominar "de múltiples oficios", el cual desempeñaba funciones muy variadas, y quien, además, se entrenaba "in situ" (característica propia de unas formas básicas para organizar el trabajo antes de que ocurriera una racionalización hecha por la administración profesional). Ese trabajador "de múltiples oficios", podía tener — como lo describe Mejía de Mesa (2014) — unos orígenes y edades particulares: "niñas y mujeres jóvenes que procedían de otras poblaciones u orfelinatos de la capital” (p. 162). Pero, sobre todo, debe tenerse en cuenta que, quizá por el papel que tenían las religiosas frente a los pacientes, el tipo de entrenamiento que recibían dichas trabajadoras se caracterizaba, además, por una amplia y compleja gama de 
deberes y funciones. Es decir, que, si las religiosas eran las personas que más entraban en contacto con los pacientes — aún más que los estudiantes e internos, quienes a la usanza de la época apenas visitaban el hospital por pocas horas-, el entrenamiento recibido y el proceso de iniciación para hacer el trabajo "artesanal", que les permitía ejecutar procedimientos médicos a este tipo de mujeres subordinadas, estaba a la orden del día. Por lo tanto, se esperaba que esas trabajadoras pudiesen llevar a cabo un rango muy amplio de tareas, desde los oficios varios de limpieza y cocina, hasta la atención a los enfermos, al menos en niveles básicos. La multiplicidad de sus actividades no solo incluían las anteriormente mencionadas, sino que también, en determinados momentos, pasaban a convertirse en camilleras y artesanas de instrumentos médicos improvisados, destinados para la intervención del cuerpo de los pacientes, en medio de unas condiciones de gran precariedad económica y frente a enormes limitaciones tecnológicas (Mejía de Mesa, 2014).

Con la llegada de la medicina de la escuela estadounidense, se abrió el camino para que se diera otro nuevo tipo de administración, con otro concepto de trabajador. A este respecto, en 1948, la Misión Humpreys visitó varias ciudades colombianas y aprovechó para criticar la situación en la que se encontraban algunos hospitales. Mención especial se hizo de la relación que deberían sostener funcionarios y empleados con lo que se esperaba tendría que ser un Hospital Universitario moderno, como el estudiado en este caso. Aquí ya se dejaba entrever el cambio en las políticas del conocimiento que llegaron al país después de la posguerra, donde Estados Unidos sentaría su preponderancia en la región (Eslava, 2002). Sin embargo, este proceso de cambio del anterior modelo de medicina francesa a la estadounidense fue, como señala Mejía de Mesa (2014), más paulatino y extendido en el tiempo de lo que podría suponerse, porque todavía en la década de los años sesenta se encontraban vestigios del primero.

El modelo estadounidense introdujo una distinción significativa con respecto a la subjetividad, puesto que cambió radicalmente las maneras de ejercer la administración y transformó la base sobre la que se construyen los trabajadores. Así, en el hospital que sigue el modelo "moderno" Flexeriano, de acuerdo con Jácome (citado por Mejía de Mesa, 2014), se presenta la nueva figura 
del director, quien a su vez ejerce la labor de administrador, acompañado de su equipo de trabajo. Se trata de una figura que profesionalmente se dedicará de forma específica a las labores administrativas. En contraste con la figura del síndico — que continuaba con la idea de la multiplicidad de tareas (o como ahora se diría "campos diferentes")—, la figura del médico-administrador se hizo acompañar de la introducción de estrategias como el organigrama, el conjunto de reglamentos para regular el trabajo, además de la retórica del trabajo en grupo.

Esos cambios no se dieron sin resistencias. Pero, con el transcurrir del tiempo, las iniciativas, recién llegadas por esa época, terminaron por darle la espalda a los modelos antiguos de medicina y de institución hospitalaria, vinculados con la escuela médica francesa, para darle paso a la visión estadounidense, que se aplicó después de la década de los sesenta, la cual surgió como resultado de un estrecho vínculo con las políticas internacionales que se implementaron para la región latinoamericana (Mejía de Mesa, 2014). Pero más que una imposición, señala Mejía de Mesa (2014), pareció darse un proceso más sutil de acogimiento de otra forma de hacer la medicina y la administración del hospital.

Hacia los ańos sesenta, y como parte del mismo proceso modernizador de este hospital universitario, también llegaron al mismo varias hermanas que procedían de otras comunidades religiosas de los Estados Unidos, dispuestas a ofrecer la atención especializada en enfermería y la docencia de esa disciplina dentro del hospital (Mejía de Mesa, 2014). Como era de esperarse, al tener esas religiosas un lugar central en las prácticas complementarias de la medicina, se llevó a cabo una transformación radical en las formas de organizar el trabajo, y en la manera como se comenzaron a concebir, por ejemplo, las tareas de las enfermeras. Si bien las hermanas de La Presentación tenían sus propias jerarquías conventuales, el manejo de la fuerza laboral típica de la enfermería llevada a cabo por ellas carecía de grandes especializaciones, lo cual se evidencia cuando una de las entrevistadas por Mejía de Mesa (2012) afirma: "todas éramos ayudantes" (p. 162). Cuando, en 1964, se incorporaron a la institución las religiosas vinculadas a otras comunidades de los Estados Unidos, comenzó a darse un desempeño laboral muy distinto, que priorizó 
elementos como la disciplina: al mismo tiempo se exigió la presentación de las trabajadoras con uniformes; se prescribió el aprecio por los horarios y las responsabilidades; así como se suspendió el ingenio y la improvisación ante ciertas emergencias, en pro de la alta valoración de los procedimientos preestablecidos, descritos y asignados a cada una, de acuerdo con su función. Esta simplificación y tecnificación del trabajo, que ocurre junto con la fundación de la escuela para profesionalizar la enfermería, llevó a que se constituyera un primer grupo de trabajadoras "entrenadas". Estas enfermeras se hallaban ahora inmersas en una red que exigía gran disciplina, la cual fue aplicada a la profesionalización como un objetivo impuesto desde las escuelas de enfermería que tenían su base en los Estados Unidos (Mejía de Mesa, 2014).

Luego, en los ańos setenta, con la llegada del modelo de la medicina preventiva, que permeaba todas las instancias del hospital universitario, se presentaron nuevamente cambios en relación con la administración del personal: se estableció una Junta Directiva propia, que de antemano desligaba al hospital de la Facultad de Medicina (Mejía de Mesa, 2012). Estos cambios, al igual que los anteriores, se enmarcaron en las disposiciones gubernamentales para la regulación de los hospitales. La situación laboral convulsionada que se presentaba en este hospital, donde el sindicato de enfermeras tuvo un papel importante, también llevó a la configuración de una oficina de personal, en condiciones de manejar los asuntos vinculados a la fuerza laboral que antes habían estado en manos de la Universidad. Ese sería también el momento en el que el modelo de consultorías especializadas empezaría a hacerse presente. Se formularían entonces una serie de nuevas prescripciones para la administración de los trabajadores. Así, en 1976, se llevó a cabo un estudio de la Facultad de Estudios Interdisciplinarios, que se propuso considerar los aspectos económicos y administrativos del hospital para hacer una serie de prescripciones que mejoraran su funcionamiento (Pérez et al. 1976). Este estudio, pionero en su género, proponía introducir la administración por objetivos, teniendo en cuenta las particularidades y dificultades que se presentaban en la historia misma del hospital.

$\mathrm{Al}$ igual que en otras organizaciones afines, por esta época se buscaba que la institución, para solucionar su problemas, entrara en la dinámica de tipo empresarial. En ese sentido, se concebía al hospital como un laboratorio para ex- 
perimentar las prescripciones que surgían desde la academia. Sin embargo, esa asesoría no se limitó a una mirada de los recursos humanos; por el contrario, la propuesta que formularon Pérez y sus colaboradores (1976) también incluía otros aspectos que se consideraron propios de una administración moderna, donde el manejo de la fuerza laboral solamente constituía uno de sus aspectos. Los investigadores de ese estudio hicieron propuestas para cambiar la estructura de la organización, la cual se reflejaba a través de un organigrama. En cuanto al personal, se invitaba a establecer una dirección que se encargara de todos los asuntos relacionados con la administración de los trabajadores, incluyendo aquello que Pérez y su equipo (1976) vieron como una selección "rudimentaria". Los asuntos relacionados con las negociaciones con el sindicato, a partir de ese año, tendrían asiento en el escenario de ese departamento que ahora también aplicaba el Código Sustantivo del Trabajo. Además, conviene anotar que en este estudio los investigadores se encontraron con que el sistema de personal estaba muy poco organizado, porque no se contaba con registros rigurosos de las transacciones que se llevaban a cabo con los trabajadores.

Pérez et al (1976) describen así el manejo de los recursos humanos por entonces:

El número de personas que labora en el hospital se determina más de acuerdo con el presupuesto hospitalario y no de acuerdo con las necesidades de personal de la institución.

En cuanto a la selección de personal no existe un procedimiento estructurado, pero sí algunas exigencias que son: en cuanto a capacidades: estas son exigidas de acuerdo con el cargo vacante y evaluadas por el jefe de departamento que necesita el nuevo empleado.

En lo referente a la educación mínima necesaria, se deja al jefe de departamento que solicita al empleado que evalúe al aspirante. La experiencia la evalúa el jefe de departamento poseedor de la vacante. La evaluación del personal directivo y/o personal especializado la realiza directamente la dirección del hospital. No existen programas de evaluación para el personal subalterno.

Bienestar social: estos programas no los realiza la oficina de personal sino la administración general del hospital.

Remuneración: el personal sindicalizado está amparado por la convención de trabajo con vigencia mayo del 76 . El personal no sindicalizado recibe remuneración de acuerdo con los criterios de las directivas del hospital, con el tipo de trabajo desempeńado y con la capacidad del empleado. 
Promoción: no existen programas de promoción para los empleados del hospital debidamente estructurados. Sin embargo, cuando hay la necesidad de llenar una vacante y se cuenta en la institución con el personal sindicalizado se le tiene en cuenta.

Evaluación de cargos. Al aspirante se le evalúa a la hora de empezar su trabajo, pero posteriormente no se le hace ningún tipo de seguimiento. Esto para los cargos subordinados y no profesionales, ya que la evaluación para los niveles profesionales es realizada por la junta directiva del hospital. Registros para la evaluación del personal: se consulta la hoja de vida. No se lleva ningún tipo de registro para los procedimientos de promoción, selección y rotación. Tampoco se elaboran (registros) de salarios ni de prestaciones (p. 173).

Pérez y su equipo interdisciplinar (1976) sugirieron por ese entonces una serie de estrategias que tendrían que ser implementadas. Como consecuencia, se implementó la administración contemporánea de la fuerza laboral a través de un departamento de recursos humanos, con divisiones y estrategias basadas en la visión de la psicología industrial-organizacional, desarrollada en las facultades de psicología de los Estados Unidos. Estas estrategias por supuesto van dirigidas, básicamente, a aumentar la percepción de satisfacción del trabajador con las tareas que ejecuta (Dávila Ladrón de Guevara, 2005; Hollway, 1991; Pulido-Martínez, 2011). Además, Pérez et al. (1976) describieron las distintas estrategias que se debían implementar, en todo nivel, para que se alcanzara a tener un departamento de recursos humanos óptimo, que garantizara el adecuado funcionamiento para la organización, y, además, con buenos resultados para los trabajadores. El tipo de administración del personal que se propuso conllevaba una visión particular del trabajador, la cual fue descrita de la siguiente manera:

Es puntual en su trabajo.

Le gusta cooperar.

Realiza todo el trabajo que se le ha asignado.

Tiene capacidad para dar órdenes.

Muestra interés por el trabajo.

Recibe órdenes de buen grado.

Consulta problemas del trabajo con jefes inmediatos.

Tiene trabajo del cual mostrarse orgulloso.

Trabaja con rapidez.

No comente errores. 
Es ordenado en sus elementos de trabajo.

Es cuidadoso con la elaboración de su trabajo.

Su apariencia personal es buena.

No necesita supervisión.

Se responsabiliza por su trabajo.

Es disciplinado.

Aprende fácilmente.

Es asequible y fácil de entender.

Es innovador.

Utiliza los conductos regulares de información.

Está en plan de constante superación individual.

Sus subordinados lo aprecian.

Reconoce sus equivocaciones.

Es amable con los pacientes (Pérez et al. 1976, p. 249).

La propuesta de un "trabajador psicológico" ideal, en conjunto con las prescripciones para su administración, entraba de este modo en el escenario del hospital universitario. Se produce este trabajador a través de las estrategias cuyos rasgos se enumeran y seleccionan previamente. Se trata de una forma mediante la cual, desde la academia, se buscaba contribuir a la solución de problemas de distintos tipos, incluidos los financieros, que el hospital frecuentemente había tenido que enfrentar. A ese respecto, Pérez et al. (1976) veían en su trabajo una forma de optimizar la organización, y anunciaban su propuesta como una nueva oportunidad para dejar de concebir las entidades hospitalarias de la manera tradicional, como todavía se hacía en el país, todo ello a partir de presentarlas como organizaciones deficitarias, en términos económicos.

Se trataba entonces de una administración del personal que, siguiendo las prescripciones de la psicología industrial-organizacional, recurría a variables como los sentimientos, habilidades, funciones, actitudes y aptitudes de los trabajadores de manera tal que, en su conjunto, deberían contribuir al incremento de la productividad (Hollway, 1991). Conviene anotar que esta manera de concebir al trabajador como sujeto psicológico se concentra en hacer prescripciones particulares para cada tipo de personas y, simultáneamente, incluye preceptos para que los trabajadores puedan ser homogeneizados, sin importar su profesión bajo la figura del empleado (Hollway, 1991; Pulido Martínez 
\& Carvajal Marín, 2016; Townley, 1994). Así, en ese amplio grupo, caben perfectamente la enfermera, el camillero, la secretaria y la persona encargada del aseo o la cocina. En esta modalidad se constituye al trabajador en términos de una interioridad que pueda mostrar todas y cada una de las características ideales y potenciales para que contribuya a aumentar la productividad de la organización.

Pues bien, esa fue la primera asesoría que se buscó en el Hospital, antes de otras que la siguieron a lo largo de su historia. A finales de la década de los años ochenta, llegarían otras propuestas que provenían de dos consultorías realizadas por empresas multinacionales dedicadas a este oficio. Se abrió así paso a un nuevo espacio para que el trabajador psicológico, que ya había sembrado sus raíces en la institución, también entrara a ser calificado como un "técnico", con funciones claramente delimitadas. En la primera consultoría, hecha por la firma Arthur Andersen (1989), se estableció una de las estrategias centrales para la administración de las organizaciones: la Planeación Estratégica. Allí aparecieron tres elementos: la misión, la visión y los objetivos, que guiarían la administración del hospital. Unos años más tarde, la asesoría conducida por Booz, Allen y Hamilton (1994) propuso muy detalladamente los puntos centrales para la constitución de un departamento de recursos humanos. Nuevamente, las recomendaciones que se realizaron buscaban uniformizar la fuerza de trabajo a través de actividades propias de esa dependencia.

En franco contraste entre el antiguo y el moderno hospital, los consultores Booz, Allen y Hamilton (1994) suspendieron —al menos sobre el papel—el que al trabajador se le concibiera únicamente en términos financieros. Propusieron que los antiguos aspectos financieros, que en algún momento habían llegado a constituir los pilares del hospital moderno, se redujeran para ser solamente uno de los aspectos centrales de la organización hospitalaria. Estos consultores también afirmaban que iban más allá del enfoque de las relaciones humanas para el manejo de los trabajadores. Este nuevo desplazamiento implicó un paso fundamental en la composición de la subjetividad, porque apartó al trabajador de la relación exclusiva con la nómina, para comenzar a concebirlo como un ser "valorado", en los mismos términos y con los mismos parámetros que el mismo sistema que estos consultores proveía. En otras pala- 
bras, la transición propuesta por Booz, Allen y Hamilton (1994) buscó abandonar la visión sobre el trabajador fundamentalmente vinculado con aspectos financieros y de nómina, para comenzar a interpretarlo a través del ciclo psicológico organizacional, que se inicia con el reclutamiento, pasa por la retención y llega hasta su retiro de la organización. Al trabajador se le agregó una clara definición de funciones, que lo convertirían en un sujeto "psicológico tecnificado" y estandarizado, el cual debería, a partir de ese momento, responder al departamento de recursos humanos, dependencia centralizada que quedaría a cargo de la estandarización y la delimitación de las funciones, limitando la injerencia que, con respecto al manejo del personal, tenían otras profesiones, como médicos y enfermeras.

A finales de la década de los noventa y durante la primera década del siglo XXI, la administración del personal hospitalario continuó un proceso cada vez más complejo con el uso de un arsenal de estrategias para el manejo psicológico de los trabajadores. En los informes de los estudiantes que hacían sus prácticas sobre el manejo de los recursos humanos en el hospital, aparece una nueva figura de la subjetividad que suele circular en las organizaciones para definir al trabajador como un "colaborador" lleno de potencialidades y dispuesto siempre a ofrecer un buen servicio de salud (Duarte \& Higuera, 2014). A esta figura se la constituye, de manera paradójica, como una manera de ser y estar en el trabajo, que busca en el mejor de los casos difuminar, en términos de apelar a la interioridad, la condición de trabajo asalariado que conlleva derechos y deberes, así como la subordinación a los requerimientos del empleador, y en el peor de los casos sirve a los procesos de flexibilización y precarización del trabajo, construyendo el trabajo como un asunto de voluntades, con el propósito de hacer las tareas en conjunto.

El trabajador definido ante todo como un colaborador y servidor emerge fundamentalmente en referencia a las nuevas herramientas que provienen de la psicología, como la administración por competencias, que busca asegurar las potencialidades de los trabajadores al servicio de la institución, así como flexibilizar algunas de las condiciones laborales. Por supuesto, esta manera de administrar el personal está enmarcada en los cambios referidos por la Ley 100 de 1993, que transformó radicalmente la manera como se ofrecía el servicio 
de salud en el país y reestructuró el papel que juegan tanto el personal médico como los usuarios del sistema de salud. El modelo de competencias establecía, en sus propios términos, una serie de niveles que deberían interactuar en beneficio de la organización, de los departamentos y respecto a los trabajadores. En la actualidad, estas denominaciones de las divisiones ya se han transformado. De la estructura original de departamentos, ahora se ha pasado a la concepción de los servicios. Y esa dependencia que antes era el departamento de gestión humana, ahora se conoce e identifica como la oficina de gestión humana.

\section{Conclusiones}

Este breve recorrido por las transformaciones del Hospital Universitario mostró diversas formas en las que, a lo largo de los años, se ha constituido la subjetividad de los trabajadores en esa institución. Como lo demuestran las figuras del trabajador múltiple, el aprendiz, el trabajador psicológico y el colaborador, la subjetividad de los trabajadores no es una cuestión inmutable e imperecedera, sino que más bien se constituye a través de un conjunto de aspectos en el que, ciertamente, la psicología, en su relación con el trabajo, ha jugado un papel central.

Al describir y enmarcar las figuras de la subjetividad en el contexto de las transformaciones del hospital universitario, se ha conseguido desfamiliarizar algunas afirmaciones comunes que celebran la llegada de la psicología como algo obvio y natural. De este modo, se señala que la aplicación de la psicología se vincula con aspectos de muy diversa índole y de muy variados niveles, que definitivamente no están vinculados tan solo con la validez de sus propuestas conceptuales y metodológicas.

Las figuras de la subjetividad muestran cómo lo que en determinado momento se concibe debe ser un trabajador, cómo este debe actuar, cambia de acuerdo con una constelación de relaciones entre las condiciones laborales imperantes, las prescripciones nacionales e internacionales que regulan el trabajo, el tipo de medicina pertinente y dominante, las relaciones geopolíticas del conocimiento, las disposiciones legales y jurídicas de su momento y, como 
lo señala Richards (2002), el estado de la psicología, incluyendo la disponibilidad local de profesionales de esta disciplina.

La producción de la subjetividad en el hospital universitario analizado, a partir de la relación entre las formas prepsicológicas y psicológicas, ha estado ligada con el desvinculamiento de la institución hospitalaria respecto a la caridad, lo que la ha llevado hacia una organización que se focaliza cada día más en la eficiencia de su visión empresarial. Aunque este parece ser un proceso naturalizado, los distintos aspectos que brevemente se han descrito aquí señalan el complejo proceso social e histórico que ha tenido lugar en el interior de esta institución.

Las figuras de la subjetividad no se reemplazan una a la otra; más bien, se producen unas relaciones de complementariedad y antagonismo entre ellas. Las propuestas acerca de la subjetividad en el trabajo son el resultado, se hacen posibles y a la vez contribuyen a mantener unas racionalidades de gobierno, que también se transforman a lo largo del tiempo, estableciendo organizaciones distintas del desempeño en el trabajo, y respecto al ejercicio de la medicina.

Como se señalaba al comienzo, se buscaba hacer una contribución austera al examen de las formas en las cuales la psicología gana un lugar y permite realizar ciertas acciones con respecto al manejo de la fuerza laboral. Ciertamente, se presenta una falta de investigación que centre su interés en aspectos relacionados con el papel de la psicología en la construcción de la subjetividad en el trabajo y, muy específicamente, en las condiciones laborales propias de la periferia. Es sorprendente que los psicólogos industriales y organizacionales, absortos en la dinámica gerencial, hayan hecho caso omiso de las aseveraciones que se han formulado en disciplinas sociales cercanas. Allí se ha hecho un llamado a considerar la constitución de la subjetividad como un asunto central para entender el mundo laboral actual. El capitalismo, haciendo uso de la psicología, está librando una batalla por la conquista de la subjetividad (Alvesson y Willmott, 2002; Pulido Martínez, 2017; Rose, 1996, 1999). La investigación crítica, en estas condiciones, no puede continuar evadiendo el examen de lugar y las operaciones de la psicología, cuando este conocimiento y sus prácticas derivadas tienen injerencia directa en la producción del sujeto 
laboral y, por lo tanto, en las maneras mediante las cuales se ejerce el gobierno de la fuerza de trabajo alrededor del mundo. 


\section{REFERENCIAS}

Alveson, M. \& Willmott, H. (2002). Identity Regulation as Organizational Control. Producing the Appropiate Individual. Journal of Management Studies, 39(5), 621-644.

Andersen \& Cia. (1989). Plan estratégico. Bogotá.

Arndt, B. \& Bigelow, M. (2013). Toward the Creation of an Institutional Logic for the Management of Hospitals: Efficiency in the Early Nineteen Hundreds. Medical Care Research and Review, 63(3), 369-394.

Baritz, L. (1960). The Servants of Power. A History of the Use of Social Science in American History. Middletown: Wesleyan University Press.

Bendassolli, P. F. (2013). Critica as apropiaçoes psicologicas do trabalho. Psicologia \& Sociedade, 23(1), 75-84.

Bernardo, M. H., Oliveira, F., Sousa, H. A. \& Sousa, C. C. (2017). Linhas paralelas: as distintas aproximaçoes da psicologia em relaçao ao trabalho. Estudos en Psicologia (Campinas), 34(1), 15-24.

Blowers, G., Cheung, B. T. \& Ru, H. (2009). Emulation vs Indigenization in the Reception of Western Psychology in Republican China: An Analysis of the Content of Chinese Psychology Journals (1922-1937). Journal of the History of the Behavioral Sciences, 45(1), 21-33. doi:10.1002/jhbs.20347

Booz, E., Allen, J. L. \& Hamilton, C. (1994). Mejoras en las Actividades de Recursos Humanos. Hospital Universitario San Ignacio. Bogotá.

Bramel, R. \& Friend, D. (1981). Hawthorne, the Myth of the Docile Worker and Class Bias in Psychology. American Psychologist, 36(8), 867-878. 
Brock, A. (2006). Internationalizing the History of Psychology. Nueva York: New York University Press.

Castaño, D. \& Sánchez, G. (1978). Problemas de la importación de la tecnología psicolaboral en los países en desarrollo. Revista Latinoamericana de Psicología, 10(1), 71-82.

Consejo Directivo de la Facultad de Medicina. (1942). Estatutos del Hospital San Ignacio de Bogotá. Bogotá.

Coutinho, M. C., Borges, R. C., Graf, L. P. \& Da Silva, A. S. (2013). "Todo dia en uma casa diferente": entre trajetórias, sentidos e o cotidiano laboral de diaristas. Universitas Psychologica, 12(4), 1125-1138.

Crespo, E. \& Serrano Pascual, M. A. (2013). La psicologización del trabajo: la desregulación del trabajo y el gobierno de las voluntades. Teoría y Critica de la Psicología, 2, 33-48.

Danziger, K. (1997). Naming the Mind. How Psychology Found its Language. Londres: Sage.

Danziger, K. (2006). Comment. International Journal of Psychology, 41(4), 269-275.

Dávila Ladrón de Guevara, C. (2001). Teorias Organizacionales y Administración. Enfoque Crítico. Bogotá: McGraw Hill.

Duarte, D. C. \& Higuera, M. (2014). Procesos de selección y capacitación en un hospital universitario. Análisis crítico (Tesis Pregrado). Pontificia Universidad Javeriana, Colombia.

Eslava, J. C. (2002). Hospital universitario y crisis hospitalaria en Colombia. Revista Gerencia y Políticas de Salud, 2, 41-48.

Fernández Ríos, M. (1995). La psicología organizacional en una encrucijada tecnológica y cultural. Revista de Psicología del Trabajo y las Organizaciones, 11(31), 49-76.

Flórez Flórez, J. (2002). Desencuentros con la psicología desarrollista. Heteropia, $8(21), 55-87$.

Foucault, M. (2006). Seguridad, Territorio y Población. Curso en el College de France (1977-1978). México: Fondo de Cultura Económica.

García Álvarez, C. M. (2008). Subjetividades laborales: impacto de la reforma de la salud en trabajadores de tres hospitales públicos en Bogotá. Pre-Til, 28, 28-44.

García Ramos, T., Báez Lebrón, M., Díaz Juarbe, R. \& Santiago Estrada, S. (2014). 
La nueva Psicología del trabajo y las organizaciones en Puerto Rico: cuatro estudios recientes. En J. Orejuela, Psicología de las Organizaciones y del Trabajo: apuestas de investigación (pp. 97-130). Cali: Editorial Bonaventuriana.

Gorbach, F. \& López, C. (eds.). (2008). Saberes locales. Ensayos sobre historia de la ciencia en América Latina. Michoacán: El Colegio de Michoacán.

Gordon, M. E., Kleiman, L. S. \& Hanie, C. A. (1978). Industrial-organizational psychology: "Open thy ears O house of Israel". American Psychologist, 33(10), 893-905. doi:10.1037/0003-066X.33.10.893

Harris, C., Cortvriend, P. \& Hyde, P. (2007). Managing people and performance: an evidence based framework applied to health service organizations. Journal of Health Organization and Management, 21(4/5), 448-459.

Henriques, J., Hollway, W., Urwin, C., Venn, C. \& Walkerdine, V. (1998). Changing the Subject. Psychology, social regulation and subjectivity. Londres: Routledge.

Hespanhol Bernardo, M. (2014). Produtivismo e precariedade subjetiva na universidade publica: desgaste mental dos docentes. Psicologia \& Sociedade, 26(n. ${ }^{\circ}$ spe.), 129-139.

Hollway, W. (1991). Work Psychology and Organizational. Managing the Individual at Work. Londres: Sage.

Holmer, M. (1997). Constructing Paper Dolls: The Discourse of Personality Testing in Organizational Practice. Communication Theory, 7(3), 189-218.

Hospital Universitario San Ignacio. (2014). Documento de Planeación Estratégica. Bogotá.

Huszczo, G. E., Wiggins, J. G. \& Currie, J. S. (1984). The Relationship between Psychology and Organized Labor: Past, Present, and Future. American Psychologist, 39(4), 432-440.

Lamal, P. A. (1991). Psychology as a Common Sense: The Case of Findings Concerning Work, Motivation and Satisfaction. Psychological Science, 2(2), 129-130.

Louw, J. (2006). Creating Subjectivity in Unexpected Places. En A. Brock, Internationalizing the History of Psychology (16-33). New York: New York University Press.

Marcon, C., Luna, I. J., \& Lucrecia, L. M. (2004). O psicologo nas institucoes hospitalares: caracteristicas e desafios. Psicologia, Ciencia e Profissao, 24(1), 28-35. Obtenido de http://pepsic.bvsalud.org/pdf/pcp/v24n1/v24n1a04.pdf

Martin, E. (2007). Bipolar Expeditions. New York: Princeton University Press. 
Mejia de Mesa, M. (2012). Entre la Mutua Dependencia y la Mutua Independencia. El Hospital San Ignacio y la Universidad Javeriana. Tesis maestria. Pontificia Universidad Javeriana. Colombia

Mejía de Mesa, M. (2014). Entre la Mutua Dependencia y la Mutua independencia. El Hospital San Ignacio y la Facultad de Medicina de la Universidad Javeriana 19421990. Bogotá: Editorial Pontificia Universidad Javeriana.

Miller, P. \& Rose, N. (2008). Governing the present. Administering economic, social and personal life. Cambridge: Polity Press.

Ortiz Martínez, J. G. (2016). Hospitales universitarios en Colombia: desde Flexner hasta los centros académicos en salud. Repertorio de Medicina y Cirugía, 25(1), 50-58.

Parker, I. (2007). Revolution in Psychology. From Alienation to Emancipation. Londres: Pluto Press.

Pérez, M. J., Castro, A., Roa, I., Cepeda, A., Ramírez, M. C., Galvis, C. M. \& Acosta Trujillo, R. (1976). Estudio interdisciplinario del Hospital San Ignacio (Tesis Pregrado). Pontificia Universidad Javeriana, Colombia.

Prilleltensky, I. (1994). Morals and Politics of Psychology. Albany: State University of New York.

Pulido Martínez, H. C. (2007). Produciendo trabajadores modernos: Conocimiento psicológico y el mundo del trabajo en el Sur. Universitas Psychologica, 6(1), 2737.

Pulido Martínez, H. C. (2010). Psychological knowledge for the governance of the South. Critical Perspectives on International Business, 6(2/3), 177-189.

Pulido Martínez, H. C. (2011). Psicología y trabajo: una relación bajo examen. En B. P. Ballesteros de Valderrama \& H. Escobar Melo (eds.), Psicología y politicas públicas. Aportes desde la academia (pp. 123-142). Bogotá: Editorial Pontificia Universidad Javeriana.

Pulido Martínez, H. C. (2014). De la psicología del trabajo y de su trabajo en el sur: acercamiento a la constitución de la subjetividad en el sector del transporte urbano de pasajeros en Bogotá. En A. Stecher \& L. Godoy (eds.), Transformaciones del trabajo, subjetividad e identidades: Lecturas psicosociales desde Chile y América Latina (pp. 213-233). Santiago de Chile: RIL Editores.

Pulido Martínez, H. C. (2017a). De la relación entre la Psicología y el trabajo a la luz de sus relaciones coloniales. Estudos de Psicologia Campinas, 34(1), 5-14. 
Pulido Martínez, H. C. (2017b). De la expansión internacional de la psicología a la simultaneidad de procesos: o de la investigación sobre la subjetividad en el trabajo. En D. Pavón Cuellar (coord.), Capitalismo y psicología crítica en Latinoamérica: del sometimiento neocolonial a la emancipación de subjetividades emergentes (pp. 243-264). México: Kanankil.

Pulido Martínez, H. C. (2018). Geopolíticas del conocimiento y riesgos psicosociales: reproduciendo la subordinación a través de las tesis de grado y postgrado. Psykhe, 26(2), 1-16.

Pulido Martínez, H. C. \& Carvajal Marín, L. M. (2016). Patologización y despatologización de la ficción del "trabajador libre" en términos del riesgo psico-laboral. En A. A. Leal Ferreira, A. Molas \& J. Carrasco (orgs.), Psicologia, Tecnologia e Sociedade: Controvérsias Metodológicas e Conceituais para uma Análise das Práticas de Subjetivação (pp. 239-258). Rio de Janeiro: NAU Editora.

Pulido Martínez, H. C. \& Sato, L. (2013). ...Y entonces ¿esto de la crítica que es? Universitas psychologica, 12(4), 1355-1368.

Richards, G. (2002). The psychology of psychology. A historically grounded sketch. Theory and Psychology, 12(1), 7-36.

Rose, N. (1996). Inventing ourselves. Psychology, power and personhood. Cambridge: Cambridge University Press.

Rose, N. (1999). Governing the Soul. The shaping of the private self. Londres: Free Association Books.

Sato, L. (2007). Procesos cotidianos de organizacao do trabalho na feira libre. Psicologia e Sociedade, 19(1), 95-102.

Schwartz, S. J., Unger, J. B., Zamboanga, B. L. \& Szapocznik, J. (2010). Rethinking the concept of acculturation: Implications for theory and research. American Psychologist, 65(4), 237-251.

Sisto, V. (2009). Cambios en el trabajo, identidad e inclusión social en Chile: Desafíos para la investigación. Universum, 24(2), 192-216.

Soto, A. (2008). Flexibilidad laboral y subjetividades: Hacia una comprensión psicosocial del empleo contemporáneo. Santiago de Chile: LOM.

Staeuble, I. (2005). The international expansion of psychology: cultural imperialism or chances for alternative cultures of knowledge. En A. Gulerce, A. Homesiter, I. Staeuble, G. Saunders, \& J. D. Kay, Contemporary Theorizing in Psychology: Global Perspectives. Concord: Captus University Publications. 
Stecher, A. (2014). El campo de investigación sobre transformaciones del trabajo, identidades y subjetividad en la modernidad contemporánea. Apuntes desde Chile y América Latina. En A. Stecher \& L. Godoy (eds.), Transformaciones del trabajo, subjetividad e identidades: Lecturas psicosociales desde Chile y América Latina. Santiago de Chile: RIL Editores.

Townley, B. (1994). Reframing Human Resources Management: Power, Ethics and the Subject at Work. Londres: Sage.

Townsend, K. \& Wilkinson, A. (2010). Managing under pressure: HRM in hospitals. Human Resources Management, 20(4), 332-338.

Ward, S. C. (2002). Modernizing the Mind. Psychological Knowledge and the Remaking of Society. Westport: Praeger.

Yoon, E., Chang C., T., Kim, S., Clawson, A., Cleary, S. E., Hansen, M., Bruner, J. P. Gomes, A. M. (2013). A Meta Analysis of Acculturarion/Enculturation and Mental Health. Journal of Counselling Psychology, 60(1), 15-30.

Mejia de Mesa, M. (2012). Entre la mutua dependencia y la mutua independencia. El Hospital San Ignacio y la Universidad Javeriana. (Trabajo de grado). Pontificia Universidad Javeriana. Bogotá. 